\title{
Burnout in der Praxis
}

Ein 50jähriger Psychiater hat sich zur Verfügung gestellt, um über den eigenen Burnout zu sprechen. Seinen Wunsch, anonym zu bleiben, respektieren wir selbstverständlich.

Wann ist Ihnen bewusst geworden, dass Sie unter einem Burnout-Syndrom leiden? Es kam schleichend. Ich habe mich zuerst lange mit vordergründigen Erklärungsversuchen zufriedengegeben, wie "ich habe mich übernommen», "ich habe die Freizeit zuwenig ausgenützt» oder «ich habe im Moment eine schwierige Klientel». Erst vor zwei Jahren etwa habe ich mich gefragt, ob nicht etwas Ernsthafteres vorliegt.

\section{Was haben Sie erlebt?}

Vor allem eine grosse Müdigkeit und eine gefühlsmässige Erschöpfung. In unserem Beruf ist das Zurverfügungstellen der Person das wichtigste Arbeitsinstrument, und genau dieses Instrument hat nicht mehr richtig funktioniert. Das Mitschwingen, die Fähigkeit, empathisch zu arbeiten, hat nachgelassen. Die Abgrenzung, welche notwendig ist, um den Beruf kunstgerecht auszuüben, ist immer schwerer gefallen. Für einen Psychiater ist das eine Katastrophe.

\section{Wurden Ihnen die Patienten gleichgültig?}

Das ist das erste, was ich gemerkt habe. In schwierigen Situationen kam der Gedanke: Nein, nicht schon wieder, ich kann nicht mehr, das interessiert mich nicht. Leute, die diesen Beruf wählen, bringen einen gewissen Idealismus mit und mögen die Menschen eigentlich. Deshalb ist Gleichgültigkeit der Horror in unserem Beruf.

Wie sah dann der berufliche Alltag aus?

Man überlegt sich Tricks, wie man den Tag überlebt. Der Druck kommt von allen Seiten: die Existenzsicherung, die Zuweisungen, die Verpflichtungen gegenüber den Hausärzten, aber auch die moralische Verpflichtung gegenüber den Patienten, die sich selbst anmelden. Man kann einen suizidalen Patienten am Telefon nicht einfach abweisen. In solchen Situationen hatte ich zunehmend das Gefühl, jetzt mag ich nicht mehr, es ist zu viel, es überschwemmt mich.

Wie haben Sie darauf reagiert?

Ich habe viel gelesen, Seminare besucht, die üblichen Schutzmassnahmen befolgt, wie Supervision, Intervision, was ohnehin selbstverständlich ist. Das genügte aber nicht mehr. Eine Teilzeitarbeit kam wegen der familiären Verpflichtungen nicht in Frage. Der Zuweisungsdruck verhindert ebenfalls eine Arbeitszeitreduktion: Es ist enorm schwierig zu sagen, so jetzt ist der Terminkalender voll. Was tun, wenn ein
Patient mit riesigen Problemen am Telefon ist, möglicherweise suizidal ist und einen Helfer braucht, aber bereits bei drei anderen Psychiatern vergeblich um einen Termin gefragt hat? Dann nein zu sagen, ist sehr schwer und auch ethisch nicht vertretbar. Es bleibt oft eine theoretische Frage, sich nach seinen Kräften richtig einzuteilen. Abwechslung und Bewegung in der Freizeit wären ebenfalls wichtige Massnahmen. Psychiater sitzen ja den ganzen Tag. Wenn aber die Erschöpfung einmal da ist, ist es ausserordentlich schwierig, zu Hause dann noch die Energie $\mathrm{zu}$ finden, sich das zuliebe zu tun, was man eigentlich brauchen würde.

Man kommt also aus einem Burnout-Syndrom nicht aus eigener Kraft heraus?

Das war bei mir eindeutig so.

\section{Wie ging es dann weiter?}

Mit einem mühsamen sich Herausarbeiten: die Praxis umstrukturieren, die Terminplanung neu festlegen, Fragen der Existenzsicherung klären, Absprachen in der Partnerschaft treffen. Es braucht ein Mittragen der Partnerin und der Familie. Wenn erste Frustsituationen auftreten, entsteht die Bestrebung, sich mit anderen Freizeittätigkeiten aufzufangen. Dabei läuft man Gefahr, sich erneut einem Energieverschleiss auszusetzen. Burnout hat meines Erachtens auch damit zu tun, dass man zuerst die Flucht nach vorn ergreifen möchte, weil man denkt, sich nicht mehr richtig zu genügen.

\section{Also setzt man einen drauf!}

Richtig, und das versucht man natürlich nicht nur in der ärztlichen Tätigkeit, sondern auch mit einer kompensierenden Freizeitbeschäftigung. Plötzlich merkt man aber, dass man sich überall verrannt hat, alles ist zuviel und frisst nur noch an der Energie. Alles muss längerfristig wieder abgebaut werden.

Hat Ihre Familie gemerkt, dass bei Ihnen etwas nicht stimmt?

Die Familie hat gemerkt, dass ich auf Distanz gehe und nicht mehr genügend verfügbar bin.

Wie hat sie reagiert, als Sie sich entschlossen haben, etwas zu verändern?

Die Familie hat die Veränderungen um meiner Gesundheit willen mitgetragen. Einschränkend war aber die kostenintensive Ausbildungszeit der Kinder. Bei ihnen wollte ich die Redimensionierung meiner Arbeitstätigkeit eigentlich nicht ansetzen.

\section{Eine Komponente des Burnout-Syndroms}

soll das Phänomen der Depersonalisation sein.

Haben Sie das auch feststellen können?

Das Sich-nicht-mehr-zur-Verfügung-stellen-Können, das Sichherausnehmen hat bis zum fast zynischen Erleben von Situationen geführt. Das hat mir Angst gemacht und mir das Gefühl gegeben, ich sei nicht mehr ich. 
Gibt es anerkannte Behandlungsansätze

für das Burnout-Syndrom?

Es ist wichtig, wenn man einen Partner hat, der hilft, aus der Situation herauszukommen und die Aussenperspektive vermittelt. Eine Behandlung soll aufdecken, eine Begleitung und eine Stütze anbieten. Weil das Burnout-Syndrom in Richtung depressiven Erlebens gehen und zu Hoffnungslosigkeit führen kann, ist Hilfe sicher angebracht.

Wie geht es Ihnen heute?

Ich bin in der letzten Phase des Verarbeitens und bin froh um dieses Interview. Es gibt mir die Gelegenheit, mir nochmals Gewissheit zu verschaffen, dass eine solche Situation nicht mehr auftreten darf. Burnout ist nicht unbedingt eine einmalige Geschichte. Das muss mich zur Vorsicht mahnen, nicht erneut in das gleiche Fahrwasser zu geraten.

Was hat bei Ihnen zum Burnout geführt?

Haben Sie eine Prädisposition für dieses Syndrom? Spielen externe Faktoren eine Rolle?

Ich habe eine grosse Bereitschaft, mit Empathie zu arbeiten. Vielleicht ein Helfersyndrom. Die Fähigkeit, emphatisch zu arbeiten, macht auch die Qualität des therapeutischen Prozesses aus. Das Hinterfragen gehört zur täglichen Arbeit. Dabei stellt sich immer die Frage, wo die Grenze zu ziehen ist zwischen dem Zurverfügenstellen der eigenen Persönlichkeit und der Abgrenzung. Das ist eine permanente Gratwanderung. Entscheidend ist die Menge der Patienten täglich fünf, sechs, sieben Patienten - und die Einseitigkeit der Arbeit über Jahre hinweg.

\section{Sind Sie auf ein spezielles Krankheitsbild spezialisiert?}

Nein das bin ich nicht. Eine Strategie, um Burnout zu vermeiden, ist, eine möglichst vielfältige Klientel zu erhalten. Nach zwei, drei depressiven Patienten hintereinander müsste man aufhören, denn dann ist wirklich genug. Wichtig ist auch, mehrere Therapiemöglichkeiten zu beherrschen.

\section{Welche Rolle spielt die ökonomische Seite des Berufs?}

Die ökonomische Seite ist für mich insofern nicht relevant, als ich mit einem bescheidenen Einkommen leben könnte. Aber es müsste auch reichen, wenn ich aus Überlastungsgründen reduzieren möchte. Hier aber ist der Engpass. Bei 1100, 1200 Patientenstunden pro Jahr erreiche ich ein durchschnittliches Einkommen von netto Fr. 10 000.- im Monat. Dies entspricht etwa dem Einkommen eines Sekundarlehrers. Wenn ich zum Beispiel auf 50\% reduzieren würde, müsste ich erhebliche Einbussen in Kauf nehmen.

Welche Rolle spielt die Anerkennung, die der Beruf des Psychiaters geniesst - oder eben nicht geniesst? Das ist auch ein wichtiger Faktor. Allein unsere Stellung innerhalb der Ärzteschaft ist eine besondere. Wir fühlen uns immer noch als Minderheit - z.B. in den Tarifverhandlungen. Unser Beitrag im Gesundheits- wesen hat einen relativ schlechten Status. Das setzt manchmal erheblich zu. Ich erlebe oft, dass Patienten sich durchs Hintertürchen in die Praxis schleichen oder auf der Strasse einen Bogen um mich herum machen. Es ist mir aufgefallen, dass bei Todesanzeigen oft dem Hausarzt für die gute Behandlung gedankt wird. Ich habe dagegen noch nie erlebt, dass einem Psychiater für die vielleicht auch jahrelange Betreuung gedankt würde. Als meine Frau einem Freund erzählte, ihr Mann habe, nachdem er Arzt geworden sei, zur Psychiatrie gewechselt, wurde ihr offen ein Bedauern ausgesprochen: "Das ist aber schade, jetzt hätte er doch ein richtiger Arzt werden können».

Sie geben dem, was Sie erlebt haben, den Namen "Burnout". Ist aber Burnout wirklich eine pathologische Entität? Gab es das nicht schon immer unter anderen Namen - oder ist das BurnoutSyndrom tatsächlich ein neues, zeitgenössisches Phänomen? Gewisse sagen, Burnout sei nur ein Modeausdruck.

Von meiner Arbeitsmethode her neige ich auch dazu, Etikettierungen zu vermeiden, weil sie sehr gefährlich sein können. Der Ausdruck kann helfen, dass sowohl Betroffene als auch Menschen um den Betroffenen herum anerkennen, dass es einen solchen Zustand gibt. Dann hat diese Etikette einen Sinn.

\section{Wie kann man ein Burnout vermeiden?}

Der einzelne muss aufpassen. Aber auch die Gesellschaft sollte in die Verantwortung genommen werden. In Berufen, die mit Menschen zu tun haben, sollte es Möglichkeiten geben, wenigstens zeitweise Alternativen zu finden oder auch umsteigen zu können. Es gibt ja typische Sackgassenberufe. Ich bin von der Allgemeinen Medizin zu weit weg, als dass ich noch eine Allgemeinpraxis betreiben könnte. Wozu kann ich noch gebraucht werden? Bin ich ein Fachidiot geworden? Muss ich den Beruf bis zur Pensionierung durchstehen? Diese Fragen stehen während eines Burnouts im Vordergrund und sind sehr quälend. Es gibt Psychiater, die im Pensionierungsalter wegen ungenügender Absicherung weiterarbeiten müssen. Es ist also auch mit 65 noch nicht unbedingt durchgestanden. Das sind niederdrückende Momente.

\section{Gibt es Ihrer Kenntnis nach Modelle,}

die hier Lösungen anbieten?

Vom Kollektiv erwarte ich mindestens Verständnis, dass das Erleben eines Burnouts nicht ein Zeichen der Schwäche ist, und dass es nicht ein Versagen ist, wenn man seinen Beruf nicht mehr schafft. Ich habe immer mit viel Neid auf den Bildungsurlaub der Lehrer geschaut. Wir Psychiater hätten es auch zugute, während eines halben Jahres einmal etwas ganz anderes zu tun oder sich Zeit zu nehmen für eine intensivere Weiterbildung. Das wären Heilmittel. Es wäre eine Herausforderung für das Kollektiv, die medizinische Karriereplanung als gemeinsames Problem anzugehen. Jungen Kollegen sollte man schon in der Weiterbildungszeit etwas mitgeben, damit sie auf solche Situationen vorbereitet sind. 
Wissen Sie von anderen Kollegen, die in einer ähnlichen Situation sind?

Das ist eine ausserordentlich wichtige Frage. Wenn man betroffen ist, ist die Versuchung gross, an sich selbst zu zweifeln. Diesen Selbstzweifel trägt man nicht gern nach aussen. Ich habe mich immer mit Neugierde gefragt, wer aus meinem Kollegenkreis auch an einem Burnout leiden könnte. Leider ist es sehr schwierig, dies herauszufinden.

$\mathrm{Zu}$ wissen, dass man nicht alleine ist mit seinem Problem, würde weiterhelfen.

Mit Sicherheit ja.

\section{Was könnte auf standespolitischer Ebene} getan werden?

Es wird viel Standespolitik betrieben, und man kann sich auch bei vielen Gelegenheiten treffen. Vielleicht sollte man bei diesen Gelegenheiten auch über persönliche Dinge sprechen und nicht immer über den Tarif. Nehmen Sie folgendes Beispiel: Die Swissair will gute Piloten und bietet ihnen deshalb eine gute Ausbildung an und zahlt einen ausgezeichneten Lohn. Mit fünfzig werden die Piloten aus dem Verkehr genommen und ihnen wird die Zeit finanziell abgegolten oder sie bekommen einen anderen Job. Wir Psychiater sollten vergleichbare Möglichkeiten haben, z. B. nach fünfzehn oder zwanzig Jahren in der freien Praxis an eine Institution zurückzugehen oder in einem wiederkehrenden Turnus verschiedene Aufgaben im Sozialbereich übernehmen zu können. Das könnte sich auch gesundheitsökonomisch positiv auswirken. Ein motivierter Psychiater leistet bessere Arbeit. Ein Erfahrungsaustausch zwischen Spital und Feld-Wald-Wiesen-Psychiatrie wäre auch eine gegenseitige Bereicherung.

Wie gross ist wohl das Verständnis für solche Vorschläge in der Ärzteschaft selbst?

Das Verständnis, wie unser Arbeitsalltag aussieht, ist oft nicht sehr gross. Hausärzte sagen etwa «Du hast fünf, sechs, sieben Patienten im Tag, das ist ja Luxus! Ich habe vierzig, fünfzig, warum klagst du? Ich hab doch mehr Grund, ausgebrannt zu sein als du". Es braucht besseres gegenseitiges Verständnis dafür, dass ein Praxisalltag mit fünf, sechs Patienten wirklich ausgelastet sein kann und dass eine zusätzliche Zuweisung nicht nur eine Notfallsituation abdeckt, sondern dass daraus oft ein therapeutischer Fall entsteht, der über Monate eine regelmässige Betreuung braucht. Gleichzeitig erleben wir den Absentismus. Wenn ein Patient nicht kommt, reisst das eine Lücke von 20\% in der Tagesplanung. Dass wir nicht so gut planen können, gehört auch zu den Stresssituationen. Schliesslich bedeutet eine Reduktion des Arbeitspensums, sich aus bestehenden Therapieverträgen rauszuschleichen. Dies kann sehr lange dauern, das bringt man innerhalb eines Jahres kaum zustande. Es gibt natürlich auch Praxen, die ganz anders organisiert sind und auf anderen Therapiemethoden aufbauen. Da sieht der Alltag wesentlich anders aus.

Wir leben in einer Zeit, in welcher berufliche Flexibilität und Freizeit zelebriert werden. Kommt man sich in einem Beruf, der so wenig davon bietet, ein bisschen dumm vor?

In kaum einem anderen Beruf wird so viel Zeit für die Weiterbildung investiert. Es braucht weitere 10 bis 15 Jahre, um die Praxis aufzubauen. Es ist dann sehr schwierig zu sagen, jetzt gehe ich, ich mache etwas ganz Neues, ich muss auf eigene Kosten mit einer neuen Ausbildung beginnen. Was macht die Familie in der Zwischenzeit? Hat sie Verständnis wie für den Bruder Klaus? Dann ist da auch eine moralische Frage: Ärzte sind doch für den Patienten da, sie dürfen nicht einfach weggehen und wegschauen. Das spielt übrigens auch in der Praxis immer noch eine wichtige Rolle. Da ist schliesslich diese doppelschichtige Haltung, welche die Gesellschaft dem Psychiater gegenüber bringt: Du musst mir helfen, wenn ich krank bin; aber wenn ich dich gebraucht habe, dann wäre mir am liebsten, es gäbe dich gar nicht.

Besten Dank für das Gespräch!

Interview: M. Trutmann 